\title{
EVALUACIÓN ANALÍTICA PARA LA DETERMINACIÓN DE SULFATOS EN AGUAS POR MÉTODO TURBIDIMÉTRICO MODIFICADO
}

\author{
Carlos A. Severiche \\ Aguas de Cartagena SA ESP \\ cseveriches@gmail.com
}

\author{
Humberto González \\ Aguas de Cartagena SA ESP \\ hgonzalez@acuacar.com
}

(Tipo de Artículo: Investigación. Recibido el 01/10/2012. Aprobado el 10/12/2012)

\begin{abstract}
RESUMEN
La meta de un análisis químico de aguas es generar resultados correctos y confiables, siendo la validación de ensayos uno de los aspectos más importantes para conseguir este propósito; además constituye un factor clave para la prestación de servicios analíticos. La determinación de ion sulfato en aguas es una de las metodologías analíticas más discutidas que se conoce en el ámbito científico técnico del análisis de aguas, principalmente, por las desventajas que presentan los métodos aceptados internacionalmente (gravimétrico, turbidimétrico y cromatográfico). En el presente estudio se hizo la evaluación del método analítico turbidimétrico, para la determinación de sulfatos en aguas; el objetivo de este trabajo fue incluir una modificación al método estandarizado y confirmar correctamente la aplicación del método modificado para el análisis de aguas. Se trabajaron muestras de diferentes tipos de agua: potable, residual, superficial, de pozo y de piscina, siguiéndose estrictamente los protocolos de validación. Se encontraron resultados satisfactorios en precisión y exactitud con el fin de emitir resultados confiables y reales de la muestra analizada.
\end{abstract}

Palabras Clave

Agua, método turbidimétrico, sulfato, validación.

\section{ANALYTICAL EVALUATION FOR THE DETERMINATION OF SULFATE IN WATER BY MODIFIED TURBIDIMETRIC METHOD}

\begin{abstract}
The goal of a chemical analysis of water is to produce accurate and reliable results, being the validation of tests one of the most important aspects to achieve this purpose, it also constitutes a key factor in the provision of analytical services. Determination of sulfate ion in water is one of the most discussed analytical methodologies in the scientific-technical sphere of waters analysis, mainly because of the disadvantages of internationally accepted methods (gravimetric, turbidimetric and chromatographic). In this study was made the evaluation of turbidimetric analytical method for the determination of sulfate in water, the aim was to include a modification to the standardized method and confirm the correct application of modified method for water analysis. We have worked on samples of different water types: drinking water, waste water, shallow, pit and pool, strictly complying the validation protocols. Satisfactory results were found regarding to precision and accuracy in order to give real and reliable results for the analyzed sample.
\end{abstract}

\section{Keywords}

Water, turbidimetric method, sulfate, validation.

\section{ÉVALUATION ANALYTIQUE POUR LA DÉTERMINATION DE SULFATES DANS EAUX AVEC LA MÉTHODE TURBIDIMÉTRIQUE MODIFIÉE}

\begin{abstract}
RÉSUMÉ
Le but d'une analyse chimique d'eaux est de générer des résultats corrects et fiables, avec la validation d'essais comme une des aspects les plus importants pour obtenir cet objectif; en plus, il constitue un facteur clé pour la prestation de services analytiques. La détermination d'ion sulfate dans eaux est une des méthodologies analytiques les plus discutés qui est connu sur le milieu scientifique technique de l'analyse d'eaux, essentiellement, à cause des désavantages qu'ont les méthodes acceptés internationalement (gravimétrique, turbidimétrique et chromatographique). Dans cette étude on a évalué la méthode turbidimétrique pour la détermination des sulfates dans eaux; le but de ce travail est d'inclure une modification sur le modèle standard et de confirmer correctement l'application de la méthode modifiée pour l'analyse d'eaux. On a travaillé sur des différents types d'eau: potable, résiduelle, superficielle, de puits et de piscine, en suivant strictement les protocoles de validation. On a trouvé des résultats satisfaisants par rapport à la précision et l'exactitude pour émettre des résultats fiables et réels de l'échantillon analysé.
\end{abstract}

\section{Mots-clés}

Eau, méthode turbidimétrique, sulfate, validation. 


\section{INTRODUCCIÓN}

El aumento en la demanda de agua potable se debe al crecimiento demográfico mundial, al rápido desarrollo económico y social, a la urbanización acelerada, y a las mejoras en el nivel de vida y de los ecosistemas circundantes [1], [2].

El control de la potabilidad y la calidad del agua es muy importante, ya que éste es el medio de trasporte de todas las sustancias y compuestos tanto biológicos como fisicoquímicos [3].

Para llevar a cabo la inspección, vigilancia y control, es necesario realizar un seguimiento de las características fisicoquímicas y microbiológicas del proceso de potabilización de agua y del producto terminado, con el fin de comparar con los valores normativos [4], [5].

Los sulfatos en el agua pueden tener su origen en el contacto de ella, con terrenos ricos en yesos, así como por la contaminación con aguas residuales industriales; el contenido de estos no suele presentar problemas de potabilidad en las aguas de consumo humano, pero contenidos superiores a $300 \mathrm{mg} / \mathrm{L}$ pueden causar trastornos gastrointestinales en los niños [6], [7]. Se sabe que los sulfatos de sodio y magnesio tienen acción laxante, por lo que no es deseable un exceso de los mismos en las aguas de consumo [8], [9].

El ion sulfato es abundante en aguas naturales. Un amplio rango de concentraciones se encuentra presente en aguas lluvias y su determinación proporciona valiosa información respecto a la contaminación y a los fenómenos ambientales; adicionalmente, puede aportar datos acerca de la información de ácido sulfúrico proveniente del dióxido de azufre presente en la atmósfera [3].

En el caso de las aguas duras, el sulfato junto con otros iones ejercen un poder incrustante y de allí la importancia de su determinación para usos industriales, especialmente en el caso de agua para calderas, ya que este fenómeno en dichos equipos, puede disminuir su efectividad y por consiguiente, su tiempo de vida [6].

En lugares donde pueda aumentar la concentración de fitoplancton, se pueden presentar zonas anaerobias debido a la descomposición de materia orgánica, en las que las bacterias afines al sulfato se activan [10], [11]. Estas bacterias toman el oxígeno de los sulfatos formando sulfuro de hidrógeno, el cual es un compuesto de olor desagradable y altamente tóxico que elimina muchos organismos del medio, excepto las bacterias anaeróbicas del ecosistema [10], [11], [12].

El ion sulfato precipita en medio ácido con cloruro de bario formando cristales de sulfato de bario de tamaño uniforme. La cantidad de cristales es proporcional a la concentración de sulfatos en la muestra y la absorbancia luminosa de la suspensión; se puede medir espectrofotométricamente a $420 \mathrm{~nm}$, siendo la concentración de $\mathrm{SO}_{4}{ }^{2-}$ determinada respecto a una curva de calibración, según los métodos normalizados para el análisis de aguas potables y residuales preparados por la Asociación Americana de Salud Pública, Asociación Americana de Trabajos del Agua, Federación para el Control de la Polución del Agua [13].

El método turbidimétrico permite determinar hasta 40 $\mathrm{mg} / \mathrm{L}$ de sulfatos. Si la muestra presenta una concentración mayor se debe realizar una dilución. Las aguas con alta turbiedad han de ser tratadas previamente por centrifugación o filtración para su clarificación y posterior análisis. Interfiere también un exceso de sílice superior a $500 \mathrm{mg} / \mathrm{L}$ y en las muestras con alto contenido de materia orgánica puede dificultarse la precipitación de sulfato de bario [10], [13].

La reglamentación Colombiana especifica los criterios y los valores respectivos para evaluar las condiciones físicas, químicas y microbiológicas de las aguas destinadas para consumo humano a través la resolución 1575 del 2007, y establece como valor máximo admisible $250 \mathrm{mg} / \mathrm{L}$ para el ion sulfato [14].

En el presente trabajo se llevó a cabo la validación del método turbidimétrico para la determinación de sulfatos en aguas. La técnica estudiada es aplicable a un rango de 1 a $40 \mathrm{mg} / \mathrm{L} \mathrm{SO}_{4}{ }^{2-}$, rango de interés, ya que la mayoría de muestras analizadas en el laboratorio están en este intervalo; se trataron los resultados de análisis obtenidos de muestras de diferente procedencia con el ánimo de hacer más completo el estudio.

\section{MATERIALES Y MÉTODOS}

El Método de referencia aplicado es el descrito en la edición 22 de los Métodos Normalizados para el Análisis de Aguas Potables y Residuales $4500-\mathrm{SO}_{4}{ }^{2-} \mathrm{E}$. APHA-AWWA-WEF (2012) [13], con la modificación soportada y desarrollada por la SOCIEDAD AMERICANA PARA PRUEBAS Y MATERIALES (ASTM 1995) D 516-90 [15]. A continuación se explican las modificaciones hechas al método original, donde de forma inicial se refiere a los reactivos donde se prepara una sola solución acondicionadora para sulfato, esta desviación con respecto a la preparación de la solución acondicionadora, se sustenta en el método ASTM (1995) [15], ya que APHA-AWWA-WEF (2012) [13], utiliza dos soluciones acondicionadoras una para el rango alto y otra para el bajo; la otra modificación tiene lugar en el procedimiento, pues la lectura a $420 \mathrm{~nm}$ se hace con celdas de $1 \mathrm{~cm}$ de paso óptico y no de $5 \mathrm{~cm}$ como plantea el método original, ya que dicho detalle haría el método más sensible; luego se tendrían que diluir buena parte de las muestras analizadas en forma rutinaria, aguas crudas de captación y especialmente, aguas potables que van a la red de distribución, haciendo el procedimiento más tedioso y complejo; hecho por el cual, se propone la modificación, debido al tipo de muestras que se analizan con frecuencia en el intervalo de 1 a $40 \mathrm{mg} / \mathrm{L}$.

Se muestra a continuación en detalle la ruta desarrollada: 
- Recolección y preservación:

Las muestras pueden colectarse en frascos de plástico o vidrio. Dado que ciertas bacterias pueden reducir el sulfato a sulfuro, especialmente en aguas contaminadas. Para su preservación se refrigera la muestra a temperatura $\leq 6^{\circ} \mathrm{C}$ y por un período máximo de 28 días.

\section{- Equipos y materiales:}

- Espectrofotómetro ultravioleta-visible UV-VIS para trabajar a $420 \mathrm{~nm}$ con celdas de $1 \mathrm{~cm}$ de paso óptico.

Vidriería: vasos de precipitados, agitadores de vidrio, volumétricos.

\section{- Reactivos:}

Para la preparación de reactivos, patrones y muestras, se empleará agua desionizada. Todos los reactivos son de grado analítico, excepto que se indique lo contrario.

Solución patrón de sulfato: utilizar solución trazable de $100-1000 \mathrm{mg} / \mathrm{L}$.

- Solución acondicionadora para sulfato: Esta se usa como forma de acondicionamiento proporcionando un medio para que los iones sulfato se mantengan en suspensión a la hora de lectura en el espectrofotómetro, es de gran importancia, ya que su no uso, precipitaría todo el analito al momento de la adición del cloruro de bario, su preparación consta de colocar en un vaso de precipitados de $1 \mathrm{~L}$ en el siguiente orden y mezclando después de cada adición: $300 \mathrm{~mL}$ de agua, $30 \mathrm{~mL}$ de ácido clorhídrico concentrado $(\mathrm{HCl}), 100 \mathrm{~mL}$ de alcohol isopropílico $\left(\mathrm{C}_{\mathrm{H}_{3}}-\mathrm{C}_{\mathrm{H}_{2}} \mathrm{OH}-\mathrm{C}_{\mathrm{H} 3}\right)$ y $75 \mathrm{~g}$ de cloruro de sodio $(\mathrm{NaCl})$. Finalmente añadir $50 \mathrm{~mL}$ de glicerol previamente medidos en una probeta. Mezclar todo y llevar a volumen final de $500 \mathrm{~mL}$ con agua. Esta solución es estable seis meses almacenada en frasco de vidrio ámbar a temperatura ambiente [15].

Cloruro de bario dihidratado $(\mathrm{BaCl} 2.2 \mathrm{H} 2 \mathrm{O})$ : Emplear una solución comercial trazable, homogeneizar antes de usar.

- Procedimiento:

Las condiciones ambientales no influyen para la realización de este ensayo.

\section{A. Preparación de la curva de calibración:}

1. Pipetear volúmenes crecientes de la solución patrón de sulfato, utilizando pipetas mecánicas previamente calibradas o de forma alternativa pipetas aforadas de vidrio, en volumétricos completar con agua desionizada hasta el aforo, para obtener al menos seis concentraciones comprendidas en el intervalo de 0 a $40 \mathrm{mg} / \mathrm{L}$.

2. Transferir los patrones a vasos de precipitado de $100 \mathrm{~mL}$. Adicionar a cada patrón $2,5 \mathrm{~mL}$ de solución acondicionadora y agitar con varilla de vidrio; adicionar una cucharilla de cristales de cloruro de bario y agitar nuevamente en forma vigorosa.

3. Leer antes de 5 minutos en espectrofotómetro a $420 \mathrm{~nm}$ con celdas de $1 \mathrm{~cm}$ de paso óptico.
4. En función del espectrofotómetro utilizado, crear la curva de calibración.

\section{B. Verificación de la curva de calibración:}

Cada vez que se analicen muestras, no es necesario construir una nueva curva de calibración, sino verificar la validez de la existente. En este caso, se prepara un patrón de concentración $20,0 \mathrm{mg} / \mathrm{L}$ y se lee como si fuera muestra. Si el resultado es coincidente $\pm 10 \%$, se considera que la curva es válida y se procede a preparar y leer las muestras [16]. En caso negativo, repetir el patrón. Si el problema persiste, verificar los reactivos, en particular, la solución madre de sulfato y, si es necesario, prepararlos y construir una nueva curva de calibración.

\section{Determinación de sulfatos en muestras:}

1. Transferir $50 \mathrm{~mL}$ de muestra (en caso de turbiedad evidente, centrifugarla 0 filtrarla) a un vaso de precipitados de $100 \mathrm{~mL}$, adicionar $2,5 \mathrm{~mL}$ de solución acondicionadora y agitar; adicionar una cucharilla de cristales de cloruro de bario y agitar nuevamente en forma vigorosa.

Leer antes de 5 minutos en espectrofotómetro a 420nm con celdas de $1 \mathrm{~cm}$ de paso óptico respecto a la curva de calibración de sulfato. Si la absorbancia de la muestra resultase mayor que la del mayor patrón, es necesario repetir el proceso mediante diluciones sucesivas de la muestra y posterior lectura en el equipo. Para esto, debe realizarse como mínimo dos diluciones, se calculó el coeficiente de variación y si éste no supera $10 \%$, se informó el valor promedio; en estos casos, es necesario multiplicar previamente por el factor de dilución [16].

\section{RESULTADOS Y DISCUSIÓN}

De acuerdo con los protocolos de validación se evaluaron los siguientes parámetros: límite de cuantificación, límite de detección, precisión, exactitud, recuperación de adiciones conocidas (exactitud en matriz) [16], [17]. Por tratarse de un método en que se modifica el paso de luz, no es necesario evaluar: identificación, selectividad, especificidad ni robustez, ya que estos parámetros no son alterados por la modificación introducida [18].

A continuación se exponen e interpretan los resultados obtenidos en los ensayos de validación del método, que se realizaron siguiendo el procedimiento de análisis referenciado. Este método fue acreditado en 2006 por el IDEAM, con base a los resultados experimentales que se presentaron y que resumidamente incluían:

\section{Exactitud:}

1. Pruebas de evaluación de desempeño del IDEAM: \# 1 de 2004 y \# 2 de 2005 satisfactorias (90 puntos en cada caso)

2. PICCAP: datos de 2004,2005 y los dos primeros envíos de 2006: satisfactorios 
3. Pruebas de añadido-recobrado: con agua potable $(n=6)$ y agua cruda $(n=7)$, recobrados de 103.8 y $102.0 \%$, respectivamente.

\section{Precisión:}

Muestra sintética de agua destilada de nivel bajo (aproximadamente $6 \mathrm{mg} / \mathrm{L}$ ), analizadas 4 réplicas durante 4 días seguidos:

Repetibilidad: $2,6-6,9 \%$ y Reproducibilidad interna: $4,7 \%$.

En la tabla 1, se muestran datos de porcentajes de coeficientes de variación promediados, para evidenciar la repetibilidad del método con muestras de diferente procedencia.

El método continuó bajo control, lo que se demuestra en los resultados satisfactorios en:

Pruebas de desempeño del Instituto de Hidrología, Meteorología y Estudios Ambientales IDEAM: 100 puntos $(2007,2008,2011), 90$ puntos (2009) y 80 puntos (2010).

Pruebas de desempeño del Instituto Nacional de Salud de Colombia PICCAP: clasificado como referencia para sulfatos durante todos estos años.

El único cambio importante ocurrió a fines de 2010, con la entrada de un nuevo analista y la sustitución del espectrofotómetro, siendo estas causales de una nueva verificación del método modificado, buscando así pruebas que evidencien repetibilidad y reproducibilidad.

\section{Tabla 1. Repetibilidad para muestras de diferente procedencia}

\begin{tabular}{|l|c|c|}
\hline \multicolumn{1}{|c|}{$\begin{array}{c}\text { TIPO DE } \\
\text { MUESTRA }\end{array}$} & $\begin{array}{c}\text { CV\% } \\
\text { promedio }\end{array}$ & $\mathbf{n}$ \\
\hline duplicados ciegas & 2,1 & 4 \\
\hline $\begin{array}{l}\text { diluciones de agua } \\
\text { de piscina }\end{array}$ & 2,4 & 4 \\
\hline $\begin{array}{l}\text { diluciones de agua } \\
\text { de pozo }\end{array}$ & 3,1 & 15 \\
\hline $\begin{array}{l}\text { diluciones de agua } \\
\text { potable }\end{array}$ & 2,5 & 79 \\
\hline $\begin{array}{l}\text { diluciones de agua } \\
\text { residual }\end{array}$ & 3,8 & 2 \\
\hline $\begin{array}{l}\text { diluciones de agua } \\
\text { superficial }\end{array}$ & 2,2 & 14 \\
\hline
\end{tabular}

Los resultados descritos se obtuvieron con muestras de agua superficial, cruda, de piscina, industrial o tratada así como con muestras certificadas y patrones de control interno.

Para las adiciones de concentración del analito se utilizaron patrones y se añadieron a muestras de agua obtenidas en la planta de tratamiento de agua potable PTAP EI Bosque: cruda del punto de llegada del agua de la captación y potable del manhole del tanque de salida. Para establecer la posible presencia de datos atípicos, se aplicó el contraste estadístico de Grubbs [16].

En las tablas 2 y 3 , se muestran datos de exactitud en patrones de referencia y añadidos recobrados en muestras respectivamente.

\section{Exactitud:}

Pruebas de añadido-recobrado:

Se realizaron por quintuplicado a muestras de agua cruda y tratada con adiciones de 4,96 y $9,92 \mathrm{mg} \mathrm{SO}_{4} / \mathrm{L}$.

\section{Con base a:}

1. El error relativo promedio de los patrones varió entre $-3,5$ y $1,1 \%$.

2. Los recobrados estuvieron entre 94,9 y $105,0 \%$ y el SM reporta recobrados entre 85 y $91 \%$ [13].

Tabla 2. Datos de exactitud para patrones

\begin{tabular}{|l|c|c|}
\hline PATRÓN & $\begin{array}{c}\text { \% Error } \\
\text { (promedio/intervalo) }\end{array}$ & $\begin{array}{c}\text { VECES } \\
\text { ANALIZADO }\end{array}$ \\
\hline $\begin{array}{l}\text { muestra } \\
\text { certificada } \\
\text { de } 72.4 \\
\mathrm{mg} \mathrm{SO}_{4} / \mathrm{L}\end{array}$ & $0,4 /-6,6 \mathrm{a} 4,0$ & 4 \\
\hline $\begin{array}{l}\text { patrón } \\
\text { interno de } \\
5 \mathrm{mg} \\
\mathrm{SO}_{4} / \mathrm{L}\end{array}$ & $1,1 /-8,3 \mathrm{a} 10,7$ & 15 \\
\hline $\begin{array}{l}\text { patrón } \\
\text { interno de } \\
20 \mathrm{mg} \\
\mathrm{SO} / \mathrm{L}\end{array}$ & $0,5 /-5,3$ a 9,0 & 28 \\
\hline $\begin{array}{l}\text { patrón } \\
\text { interno de } \\
36 \mathrm{mg} \\
\mathrm{SO}_{4} / \mathrm{L}\end{array}$ & $-3,5 /-9,2 \mathrm{a} 0,7$ & \\
\hline
\end{tabular}

Se observa que el método no presenta tendencia alguna que indique sesgos ni errores sistemáticos apreciables [19], debido a interferencias presentes en la matriz de las muestras ni al proceso de análisis mismo. Se considera satisfactoria la exactitud [16].

Tabla 3. Datos de recobrado

\begin{tabular}{|l|c|}
\hline \multicolumn{1}{|c|}{ MUESTRA } & $\begin{array}{c}\text { \% recobrado } \\
\text { (promedio/intervalo) }\end{array}$ \\
\hline $\begin{array}{l}\text { agua cruda }+4,96 \mathrm{mg} \\
\mathrm{SO}_{4} / \mathrm{L}\end{array}$ & $100,1 / 96,5-102,3$ \\
\hline $\begin{array}{l}\text { agua cruda }+9,92 \mathrm{mg} \\
\mathrm{SO}_{4} / \mathrm{L}\end{array}$ & $98,8 / 94,9-105,0$ \\
\hline $\begin{array}{l}\text { agua tratada }+4,96 \mathrm{mg} \\
\mathrm{SO}_{4} / \mathrm{L}\end{array}$ & $97,4 / 95,1-100,1$ \\
\hline $\begin{array}{l}\text { agua tratada }+9,92 \mathrm{mg} \\
\mathrm{SO}_{4} / \mathrm{L}\end{array}$ & $95,6 / 93,7-100,1$ \\
\hline
\end{tabular}

En la tabla 4, se muestran los datos de repetibilidad para muestras de diferente procedencia (aguas crudas, tratadas y piscinas). 
Tabla 4. Datos de repetibilidad

\begin{tabular}{|l|c|c|}
\hline \multicolumn{1}{|c|}{ TIPO DE MUESTRA } & CV\% & n \\
\hline agua cruda & 0,9 & 3 \\
\hline agua cruda & 4,0 & 5 \\
\hline agua cruda + añadido 1 & 2,9 & 5 \\
\hline agua cruda + añadido 2 & 3,8 & 5 \\
\hline agua tratada & 3,1 & 5 \\
\hline agua tratada + añadido 1 & 2,2 & 5 \\
\hline agua tratada + añadido 2 & 2,7 & 5 \\
\hline agua superficial & 0,1 & 3 \\
\hline agua potable de red & 1,5 & 3 \\
\hline agua potable & 1,6 & 3 \\
\hline agua de piscina & 0,3 & 3 \\
\hline
\end{tabular}

\section{Precisión:}

Análisis de muestras de diferente procedencia. Con base a los contenidos de sulfatos $(<40 \mathrm{mg} / \mathrm{L})$ y la tabla de Horwitz, puede considerarse satisfactoria la repetibilidad [19]. Para 16 muestras de diverso origen: potable (5), pozo (1), industrial (2) y desconocida (8), con contenidos entre $45-8000 \mathrm{mg} \mathrm{SO} / \mathrm{L}$, el CV promedio fue de 3.4\%, desviación estándar 0,3-9,1. En las tablas 5 y 6 , se muestran datos de reproducibilidad interna para muestras y controles internos respectivamente y el número de veces analizadas.

\section{Tabla 5. Datos de reproducibilidad interna para} muestras

\begin{tabular}{|l|c|c|}
\hline \multicolumn{1}{|c|}{$\begin{array}{c}\text { TIPO DE } \\
\text { MUESTRA }\end{array}$} & CV\% & MUESTRAS \\
\hline agua potable & $4,0-7,6$ & 2 \\
\hline agua industrial & $2,7-4,5$ & 2 \\
\hline
\end{tabular}

Para la reproducibilidad interna, se analizaron muestras dos días diferentes no consecutivos, con patrones de control interno. Y se considera también satisfactoria la precisión [19].

Tabla 6. Datos de reproducibilidad interna para patrones de control interno

\begin{tabular}{|c|c|c|}
\hline $\begin{array}{c}\text { Concentración } \\
\text { del patrón }(\mathbf{m g} \\
\left.\mathbf{S O}_{\mathbf{4}} / \mathbf{L}\right)\end{array}$ & $\mathbf{C V}^{\text {CV }}$ & $\begin{array}{c}\text { VECES } \\
\text { ANALIZADO }\end{array}$ \\
\hline 5 & 5,7 & 15 \\
\hline 20 & 3,6 & 28 \\
\hline 36 & 2,7 & 9 \\
\hline
\end{tabular}

Curva de calibración:

Intervalo de trabajo: $5-40 \mathrm{mg} / \mathrm{L}$

Coeficiente de correlación: 0.9999

CV método: $1.53 \%$

Límite de detección $\left(X_{b l}+3 S_{b l}\right): 0.53 \mathrm{mg} \mathrm{SO}_{4} / \mathrm{L}$

Límite de cuantificación $\left(X_{b l}+10 S_{b l}\right): 1.83 \mathrm{mg} \mathrm{SO} / / \mathrm{L}$

Concentración a reportar: $5.0 \mathrm{mg} \mathrm{SO} / \mathrm{L}$

Dado que la legislación colombiana para agua potable considera $250 \mathrm{mg} / \mathrm{L}$ como valor máximo aceptable para sulfatos y que la mayoría de aguas naturales o residuales, presentan concentraciones superiores a 5 $\mathrm{mg} / \mathrm{L}$, la concentración mínima a reportar [13], se considera satisfactoria para el análisis de agua, pues es 50 veces el valor máximo permisible [14].

\section{CONCLUSIONES}

El método estandarizado modificado presenta adecuadas características de desempeño, al ser preciso (coeficientes de variación $<10 \%$ ), veraz (no presenta sesgo significativo), con un adecuado intervalo de concentración y una concentración mínima a reportar, baja $(5 \mathrm{mg} / \mathrm{L})$.

Estas características permiten que el mismo se ajuste al propósito para el cual fue diseñado, que consiste en la determinación de sulfato en muestras de aguas de diferente procedencia.

El usar una sola solución acondicionadora es una ventaja en tiempo y la extensión del rango dinámico lineal hasta $40 \mathrm{mg} / \mathrm{L}$, lo cual implica maximización de análisis, en muestras con sulfatos en rangos rutinarios y habituales, con un mayor volumen de capacidad.

Se vuelve un método de referencia para otros laboratorios que deseen aplicarlo en Colombia, al estar este parámetro acreditado ante el IDEAM, ya que pueden reportar resultados correctos y confiables.

\section{REFERENCIAS}

[1] H. Cheng, Y. Hu \& J. Zhao. "Meeting China's water shortage crisis: current practices and challenges". Environm. Sci. Techn. J. (USA). Vol. 43, No. 2, pp. 240244, 2009.

[2] I. Sarabia. "Calidad del agua de riego en suelos agrícolas y cultivos del Valle de San Luis Potosí, México". Rev. Int. Contam. Ambient. (México). Vol. 27, No. 2, pp. 103-113, 2011.

[3] J. Arboleda. Teoría y práctica de la purificación del agua. Colombia: Ed. Mc Graw Hill, 2000, p.31.

[4] M. Simanca, B. Álvarez \& Paternina, R. "Calidad física, química y bacteriológica del agua envasada en el municipio de Montería". Temas Agrarios. (Colombia). Vol. 15, No. 1, pp. 71-83, 2010.

[5] EPA. Guidelines for Water Reuse, EPA- Part III, 40 CFR, Part 122, U.S. Environmental Protection Agency U.S. Agency for International Development, Washington, DC, 2007, p.136.

[6] C. Orozco, A. Pérez, M. González, F. Rodríguez \& J. Alfayate. Contaminación ambiental. Una visión desde la química. Ed. Thomson, España, 2005, pp. 631-650.

[7] D. Wilson, A. Fernández \& Y. Zayas. "Desarrollo y validación de un método de valoración anemométrica para la determinación del ion sulfato en muestras de aguas naturales y residuales". Rev. Cub. Qca. (Cuba). Vol. 19, No. 2, pp. 28-33, 2007.

[8] G. Guzmán. "Evaluación espacio-temporal de la calidad del agua del río San Pedro en el Estado de Aguascalientes, México". Rev. Int. Contam. Ambient. (México). Vol. 27, No. 2, pp. 89-102, 2011.

[9] F. Ríos; A. Maroto \& R. Bosque. "La validación de Métodos analíticos". Rev. Tec. Lab. (Cuba). Vol. 22, No. 252, pp.12-17, 2001.

[10] I. Aguilera; R. Perez \& A. Marañon. "Determinación de sulfato por el método turbidimétrico en aguas y aguas residuales. Validación del método". Rev. Cub. Qca. (Cuba). Vol. 22, No. 3, pp. 39-44, 2010. 
[11] S. Stolyar et al. "Metabolic modeling of a mutualistic microbial community". Molecular Systems Biology. Vol. 38, No. 92, pp. 1-14, 2007.

[12] P. Guevara \& M. Ortiz. “Adaptación a microescala del método potenciométrico con electrodo ión selectivo para la cuantificación de fluoruro". Rev. Int. Contam. Ambient. (México). Vol. 25, No. 2, pp. 87-94, 2009.

[13] APHA, AWWA, WEF. Standard Methods for the Examination of Water and Wastewater. 22th Ed. American Public Health Association/ American Water Works Association/Water Environment Federation, Washington DC, USA, 2012, pp. 4-188 y 4-190.

[14] MINISTERIO DE LA PROTECCIÓN SOCIAL, MINISTERIO DE AMBIENTE VIVIENDA Y DESARROLLO TERRITORIAL. Por medio de la cual se señalan características, instrumentos básicos y frecuencias del sistema de control y vigilancia para la calidad del agua para consumo humano. Resolución 1575 de 2007, Bogotá, D.C.: Ministerio de la Protección
Social; Ministerio de Ambiente Vivienda y Desarrollo Territorial, 2007.

[15] ASTM. Standard test methods for sulfate ion in water. Ed ASTM annual book, USA, 1995, D 516-90.

[16] G. Cortes. Lineamientos para el control de calidad analítica. Ed. Instituto de Hidrología, Meteorología y Estudios Ambientales IDEAM. Colombia, 1999, p.11.

[17] C. Cortés \& R. García. Validación con base en los criterios de aplicación de la norma NMX-EC-17025IMNC-2006 en mediciones químicas y físicas. Ed. Entidad Mexicana de Acreditación. México, 2009, p.25.

[18] C. Cortés. Validación de métodos. Docto. No. MP. CA005-02. Ed Entidad Mexicana de Acreditación. México, 2010, p.37.

[19] M. Velázquez, J. Pimentel \& M. Ortega. "Estudio de la distribución de boro en fuentes de agua de la cuenca del río Duero, México, utilizando análisis estadístico multivariado". Rev. Int. Contam. Ambient. (México). Vol. 27, No. 1, pp. 9-30, 2011. 